\title{
CONF- $950262-1$
}

Note: This is a preprint of a paper being submitted for publication. Contents of this paper should not be quoted nor referred to without permission of the author(s).

Kinetic Modeling of the Atomic Layer Epitaxy Window in Group IV Semiconductor Growth

\author{
Gyula Eres
}

Submitted to Optical Society of America Winter Topical Meetings

February 9-11, 1995

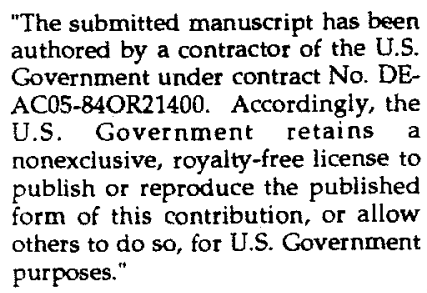

"The submitted manuscript has been authored by a contractor of the U.S. Government under contract No. DEACO5-84OR21400. Accordingly, the U.S. Government retains a nonexclusive, royalty-free license to publish or reproduce the published form of this contribution, or allow others to do so, for U.S. Government purposes."

Solid State Division

Oak Ridge National Laboratory

P. O. Box 2008

Oak Ridge, Tennessee 37831-6056 managed by

MARTIN MARIETTA ENERGY SYSTEMS, INC. for the

U.S. DEPARTMENT OF ENERGY under contract DE-AC05-84OR21400

Research sponsored by the Division of Materials Sciences, U.S. Department of Energy under contract DE-AC05-84OR21400 with Martin Marietta Energy Systems, Inc. 


\section{DISCLAIMER}

This report was prepared as an account of work sponsored by an agency of the United States Government. Neither the United States Government nor any agency thereof, nor any of their employees, make any warranty, express or implied, or assumes any legal liability or responsibility for the accuracy, completeness, or usefulness of any information, apparatus, product, or process disclosed, or represents that its use would not infringe privately owned rights. Reference herein to any specific commercial product, process, or service by trade name, trademark, manufacturer, or otherwise does not necessarily constitute or imply its endorsement, recommendation, or favoring by the United States Government or any agency thereof. The views and opinions of authors expressed herein do not necessarily state or reflect those of the United States Government or any agency thereof. 


\section{DISCLAIMER}

Portions of this document may be illegible in electronic image products. Images are produced from the best available original document. 


\title{
Kinetic Modeling of the Atomic Layer Epitaxy Window in Group IV Semiconductor Growth
}

\author{
Gvula Eres \\ Uak Ridge National Laboratory, P.O. Box 2008, Solid State Division. Bldg. 2000. MS 6056. \\ Oak Ridge, Tennessee 37831-6056 \\ Phone: (615) 574-5494, FAX: (615) 574-4814
}

Atomic laver epitaxy (ALE) is a method for epitaxial growth of semiconductor thin films that utilizes the adsorption kinetics of source gases for external control of the film growth process. 11] ALE takes advantage of the self-limiting nature of the chemisorption process for achieving laver-by-laver growth of semiconductors with uniform film thickness, ideally, $1 \mathrm{ML}$ per each adsorption cycie. The $1 \mathrm{ML} /$ cycle film growth is a uniquely distinguishing feature, that makes ALE highly attractive for preparation of ultrathin films of precisely controlled thickness. Other unique characteristics of ALE growth are that the spatial homogeneity of thin films, and the film thickness per cycle are insensitive to small variations in the external process parameters. The parameter space in which ALE is independent of external conditions is known as the ALE window. [2]

The existence of the ALE window in II-VI. and III-V compound semiconductor ALE is intuitive and fairly straightforward to realize in practice. [2.3] The polar nature of the chemical bonding between successive lavers in these materials assures that each chemisorption cycle terminates after formation of a monolayer. Multilayer adsorption characterized by much weaker interactions is easily eliminated by elevating the substrate temperature above the range where physisorption occurs. A film of finite thickness is deposited by alternating exposure to the constituent source gases in a sequential fashion.

Because bonding in group IV materials is covalent (non-polar), group IV ALE cannot be realized simply based on the difference in the interaction strength between chemisorption and physisorption. Instead, an alternative strategy that is based on utilizing bonding with peripheral atoms of different electronegativity has been proposed. [4] For example. several processes using the $\mathrm{H} / \mathrm{Cl}$ exchange reaction for realizing silicon $A L E$ have been reported. [5] Silicon $\mathrm{ALE}$ by the $\mathrm{H} / \mathrm{Cl}$ exchange reaction is perceived to occur between a saturated laver of either surface hydride, or surface chloride with the appropriate silicon source gas to release $\mathrm{HCl}$ and to deposit a silicon film. However. formation of an ALE window with either silicon hydride, or silicon chloride source gases is not well understood. [6] In this paper. the role of a fundamental kinetic parameter. the kinetic order of the elementary reaction steps, in formation of the ALE window in group IV semiconductors is explored by modeling the overall film growth reaction in terms of its elementary reaction steps. To faciitate meaningful comparison with experiments. the formation, and evolution of a hydridic laver. for which a wealth of data exist will be used as an example. [7] However. the conclusions that are reached have a general significance, and are applicable in other chemisorption systems.

The surface hydride coverage in epitaxial growth of silicon from silanes is the consequence of the dynamic equilibrium between silane chemisorption and molecular hydrogen desorption. However, instead of the absolute hydride coverage, the description of the thin film growth process is more meaningful in terms of the depletion and the regeneration of active film growth sites. [7,8] In group IV thin film growth. the dangling bonds represent the active sites. The depletion of the active surface sites is described by the following rate equation:

$$
\frac{d \theta}{d t}=k_{1}(1-\theta)^{n}-k_{2} \theta^{m}
$$

where $\theta$ is the fraction of depleted active sites. $k_{1}$ is the depletion rate constant. $k_{2}$ is the regeneration rate constant, and $n$. and $m$, are the reaction orders of the depletion, and the regeneration steps, respectively.

Hydrogen desorption from the $\mathrm{Si}(100)$ has received a great deal of attention in recent years because it was found to be first order, rather than second order as expected for recombinative desorption. [9] Additionally, first order molecular hydrogen desorption was found following chemisorption of disilane on the $\operatorname{Si}(100)$ surface. 77] According to experimental data, first order behavior of hydrogen desorption from the Si(100) sirface is quite general and is independent of whether the hydride coverage is produced by atomic hydrogen adsorption. or by chemisorption of silanes. [10] Similarly, first order hydrogen desorption was found from the Ge(100) surface following chemisorption of digermane. [11] Therefore, governed by the experimental data, the solution to the above rate equation will only be sought for a first order $(m=1)$ regeneration step. 
In contrast with molecular hydrogen desorption. a consensus has not been reached on the reaction order of the chemisorption step. The results of mecnanistic studies of fragments produced by annealing of arisorption layers of silanes tormed below room temperature. suggest that the elementary reaction step of source gas 'hemisorption is second order. [12] On the orher hand. the reaction order in kinetic studies of thin film nrowth processes was found to be first orrier. 7 Since the reaction ordier of the chemisorption step is not clearly established by experiment, both first $(n=1)$. and second $(n=2)$ order depletion of active sites need to be considerred in modeling of the film growth process.

Tile solutions to the rate equation describe the time dependence of the active site coverage. The solution tor $n=m=1$ is given by:

$$
\theta(t)=\theta_{0} \exp \left[-\left(k_{1}+k_{2}\right) t\right]+\frac{k_{1}}{\left(k_{1}+k_{2}\right)}\left\{1-\exp \left[-\left(k_{1}+k_{2}\right) t\right]\right\}
$$

where $\theta_{0}$ is the fraction of the ciepleted active sites at $t=0$. For $n=2$ and $m=1$ the solution is given by:

$$
\theta(t)=\frac{1}{2 k_{1}}\left[2 k_{1}+k_{2}+r\left(\frac{1+C \exp (r t)}{1-C \exp (r t)}\right)\right]
$$

where $r=\sqrt{k_{2}^{2}+4 k_{1} k_{2}}$, and $C^{c}=\left(2 k_{1} \theta_{0}-2 h_{1}-k_{2}-r\right) /\left(2 k_{1} \theta_{0}-2 h_{1}-k_{2}+r\right)$. In terms of the absolute hydrogen coverage, the above solutions represent pure monohvdride coverage.

Since the thin film growth rate is cirectiy proportional to the rate of the active site depletion. the total film thickness $\mathcal{T}$ at time $t$ is obtained by integrating the active site depletion rate from Eq. (1) between 0 and $t$.

$$
\mathcal{T}(\mathrm{ML})=\int_{0}^{t} k_{1}(1-\theta(t))^{n} d t
$$

The integral in Eq. (4) for $n=1$ is given bv:

$$
\left.\mathcal{T}(\mathrm{ML})=-\frac{k_{1}\left(\theta_{0}\left(k_{1}+k_{2}\right)-k_{1}\right)}{\left(k_{1}+k_{2}\right)^{2}}+\frac{k_{1} k_{2}}{\left(k_{1}+k_{2}\right)} t+\frac{k_{1}\left(\theta_{0}\left(k_{1}+k_{2}\right)-k_{1}\right)}{\left(k_{1}+k_{2}\right)^{2}} \exp \mid-\left(k_{1}+k_{2}\right) t\right) .
$$

and tor $n=2$ the integral is given by:

$$
T(\mathrm{ML})=\frac{1}{4 k_{1}}\left[k \cdot 2 k_{2} r\left(\frac{1+c^{\prime} \exp (r t)}{1-c^{\prime} \exp (r t)}\right)+r^{2}\left(\frac{1+(\cdot \exp (r t)}{1-c^{\prime} \exp (r t)}\right)^{2}\right] t
$$

Assuming that one original active site is regenerated per each atom that is incorporated into the film. Eq. (3) and Eq. (6) describe the evolntion of film thickness for first. and second order depletion of active sites. respectively.

The significance of Eq. (5) and Eq. (6) is that these equations identify time as an important variable in the ALE of group IV semiconductors. The time-dependence in group IV semiconductor growth comes from the fact that the active sites regenerated by desorption of molecular hvdrogen are immediately depleted by chemisorption of new source gas molecules. resulting in continuous film growth. However. the first term in Eq. $(5)$ is independent of time. and suggests that a self-limiting mechanism could be achieved if the contributions from the time-dependent terms are made negligible. Assuming that $\forall_{0}=0$. Eq. (5) shows that irieal ALE. i.e. $1 \mathrm{ML} /$ cycle growth requires that $k_{1}>>k_{2}$,

$$
\mathcal{T}(\mathrm{ML})=1+k_{2} t-\exp \left(-k_{1} t\right)
$$

The exponential term in the above equation is associated with reaching steady-state. and the $h_{2} t$ term describes continuous film growth. characteristic of gas source NBE. Eq. (5) and Eq. (7) illustrate that thin film growth from a first order depletion step occurs by two kineticaliv different stages. The initial stage corresponding to growth of the first monolaver can be thought to occur in a discrete tashion. After the first monolaver is grown. the rest of the film grows continuosiy. Consequently, a strategy for achiering ALE requires separation of the two film growth stages, and assuring that upon completion of the first monolayer, continuous film growth is cut off as quicky as possible. The parameter space for achieving ALE type behavior 
is illustrated by the three-dimensional plot in Fig. 1. The ALE window corresponds to the plateau region in which monolayer-thick adsorption laver formation is maintained.

In comparison, the expression for film growth from a second order chemisorption step given bv Eq. (6), contains only time-dependent terms. The time-dependence implies continuous film growth. and more importantly, indicates that no self-limiting mechanism can be generated based on the kinetic parameters considered by the model. Thus, if depletion of the active sites occurs by a second order process. ALE cannot be realized because no mechanism for turning off continuous film growth exists. A three-dimensional plot of the film thickness given by Eq. (6) is shown in Fig. 2. The film thickness shows a linear dependence on the growth parameters. except in the very early stages of growth where a transient peak is predicted. The transient peak scales with the fraction of initially covered active sites $\left(\theta_{0}\right)$. The transient peak has a maximum height for $\left(\theta_{0}=0\right)$ and disappears for $\left(\theta_{0}=1\right)$. Therefore, second order depietion of active sites could be confirmed by observation of the transient film growth peak.

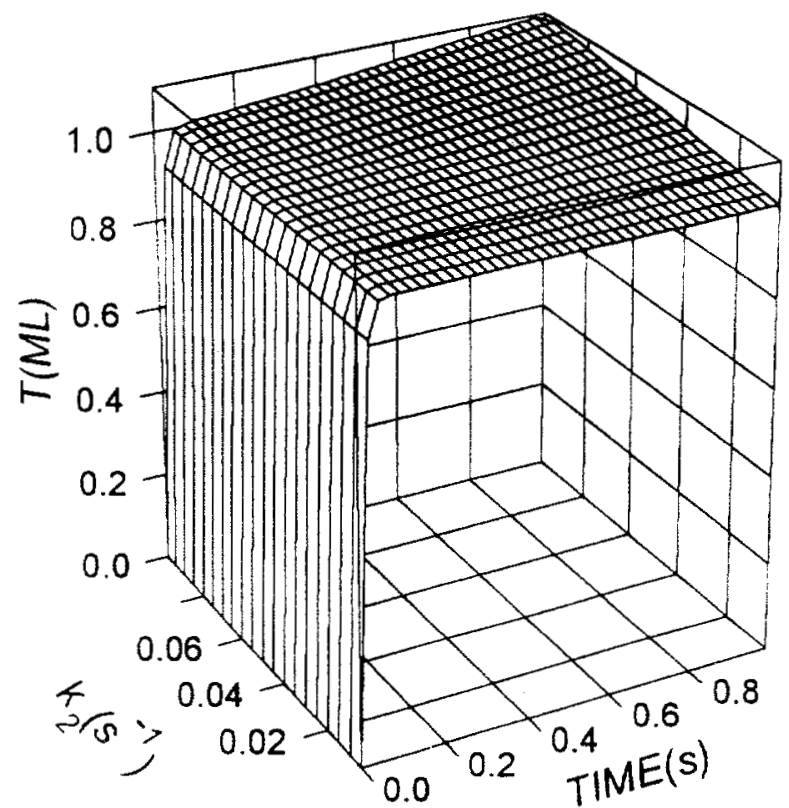

FIG.1. Three-dimensional plot of the film thickness for $n=m=1$ and a fixed $k_{1}=100 s^{-1}$. The plateau in the 1 ML region represents the ALE window.

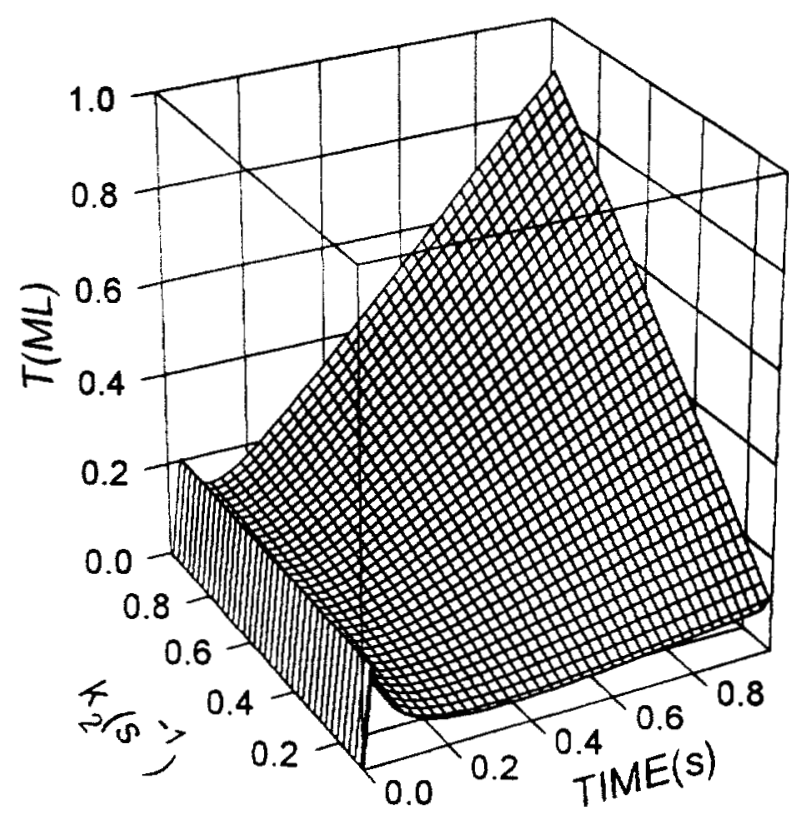

FIG.2. Three-dimensional plot of the film thickness for $n=2, m=1$ and $k_{1}=100 \mathrm{~s}^{-1}$. Note the transient peak at $0.2 \mathrm{ML}$. and the absence of self-limiting behavior.

[1] See articles in Thin Solid Films 225, (1993).

[2] T. Suntola, Mater. Sci. Rept. 4, 261 (1989).

(3) M. Ozeki, Mater. Sci. Rept. 8, 97 (1992).

4) C.H.L. Goodman and M.V. Pessa, J. Appl. Phys. 60, 65R (1986).

[5] Thin Solid Films 225, 145-212 (1993).

(6) P. Hirva and T.A. Pekkanen. Surf. Sci. 220. 137 (1989).

[7] G. Eres and J.W. Sharp, J. Appl. Phys. 74. 7241 (1993).

[8] K. Werner. S. Butzke, S. Radelaar, and P. Balk, J. Crystal Growth. 136. 338 (1994).

[9] K. Sinniah. M.G. Sherman, L.B. Lewis, W.H. Weinberg, J.T. Yates, Jr., and K.C. Janda. J. Chem. Phys. 92, $5700(1990)$

[10] K.J. Uram and U. Jansson, J. Vac. Sci. Technol. B 7, 1176 (1989).

[11] G. Eres and J.W. Sharp, J. Vac. Sci. Technol. A 11, 2463 (1993).

[12] S.M. Gates and S.K. Kulkarni, Appl. Phys. Lett. 58, 2963 (1991). 